\title{
Optimization of a parameter in laser heating process
}

\author{
Htin Linn Oo \\ Student, National Research University of Electronic Technology (MIET)
}

\begin{abstract}
The aim of this work is the parameter modeling of the metal heating process by laser heating. For these simulations COMSOL Multiphysics 5.3a is used. The transient control of the laser beam is modeled by dynamic equations in the code. The used heat transfer module of COMSOL offers two possibilities of modeling the interaction between laser beam and metal. The first one is that the laser beam is regarded as an incoming thermal flow at the metal, at the other way basically the modeling of the laser beam as a local volume heat source is on focus. The results of the modeling, that have been achieved, show a good agreement between model and investigation, being an important part of further research works.
\end{abstract}

Keywords: modeling; laser processing; simulation; aluminum

\section{Introduction}

Today, the problem of laser heating process and thermal properties investigation and complex materials are still increasing. Lasers are used in material processing to heat, to melt or to fusion exactly energy of the surface and the inner part stays without changes. The purpose of this work is to control the parameter of laser processing by COMSOL Multiphysics, in order to analysis the effects of laser power, speed, surface emissivity of material, focal point of laser and temperature of laser. For this simulation is used COMSOL Multiphysics to predict the melting energy by lasers. In this paper aluminum is assumed to be rotate on it stage and the heat transport equation is used for the energy (photons) absorption in depth. A model of laser interaction with moving material was created in COMSOL.

\section{Materials and Methods}

\subsection{Thermal Modeling}

\subsubsection{Heat Equations}

The surface of metal is dissipated by laser beam heated on it, according to conduction, convection, and radiation. The modeling can be provided by theoretical approach. So, the heat transport equation can be given as:

$$
\rho c \frac{\partial T}{\partial t}=\nabla \cdot(k \nabla T)+\alpha \downarrow
$$


where $\mathrm{T}$ is the temperature, $\mathrm{t}$ is the time, $\mathrm{k}$ is the thermal conductivity, $\mathrm{Q}$ is the laser heat generation, $\alpha$ is the absorption coefficient, $\mathrm{c}$ is the heat capacity and $\rho$ is the density . In addition, radiation and convection losses $q_{c}$ and $q_{r}$ are given as:

$$
q_{c}=h\left(T \infty-T_{\text {room }}\right)
$$

where $\mathrm{T} \infty$ and $T_{\text {room }}$ are the gas medium and room temperature, $\mathrm{h}$ is the heat convection coefficient and $\varepsilon$ is the emissivity, respectively. In the following sections, losses will be provided by domain of interest form of boundary conditions; both To and $T_{\text {room }}$ will be assumed as $20{ }^{\circ} \mathrm{C}$.

$$
q_{r}=\varepsilon\left(T_{\text {room }}^{4}-T^{4}\right)
$$

\subsubsection{Heat Source}

At firstly, need to description the fundamental mood of the laser heat generation. The fundamental mode of a Gaussian beam is usually better and a Gaussian heat source is given, accordingly. The latter is considered in this paper; namely, the heat generated by a super-Gaussian profile (i.e., a smoothed flat-top profile) of transverse optical intensity of order $n$ can be given as:

$$
Q(r)=Q_{0} \exp \left[-2\left(\frac{r}{\omega_{0}}\right)^{n}\right]
$$

where $\omega_{0}$ is the beam radius over the surface of object, $Q_{0}$ is the optimum intensity, and $\mathrm{r}$ is the radiate distance from the generation axis. In this paper was implemented by super-Gaussian intensity profile of order 20, based on actual data gain from beam profiler. Under this condition and for P indicate the loading power, the peak intensity in Equation (4) approaches:

$$
Q_{0}=\frac{P}{\square \omega_{0}^{2}}
$$

In addition, the model is purposed to modeling an application of repairing from metal where a defocused beam must be used, processing diameter of $2 \mathrm{~mm}$ was defocused by the laser beam.

\subsubsection{Material Properties}

In this model, the most important is the temperature-dependent of material, to be exactly prediction of the temperature. For this goal, based on the data literature on the properties of aluminum and its alloys, a lot of methods have been included in the background for the properties 


\section{On Applied Research In}

\section{SCIENCE, TECHNOLOGY \& KNOWLEDGE}

\section{5-17 March 2019}

involved in conduction, convection, and radiation equations. For create products is used; liquid and solidus temperature of 775 and $911 \mathrm{~K}$, respectively, are given.

At firstly, a functional form for density $\left(\mathrm{kg}_{\mathrm{g}} \mathrm{m}^{-3}\right)$, depending on the accumulation status:

$$
\rho=\theta_{\text {solid }} \rho_{\text {solid }}+\left(1-\theta_{\text {solid }}\right) \rho_{\text {liquid }} \quad 775<\mathrm{T}<911 \mathrm{~K}
$$

Figure 1: The sequence of the control parameters of laser processing 


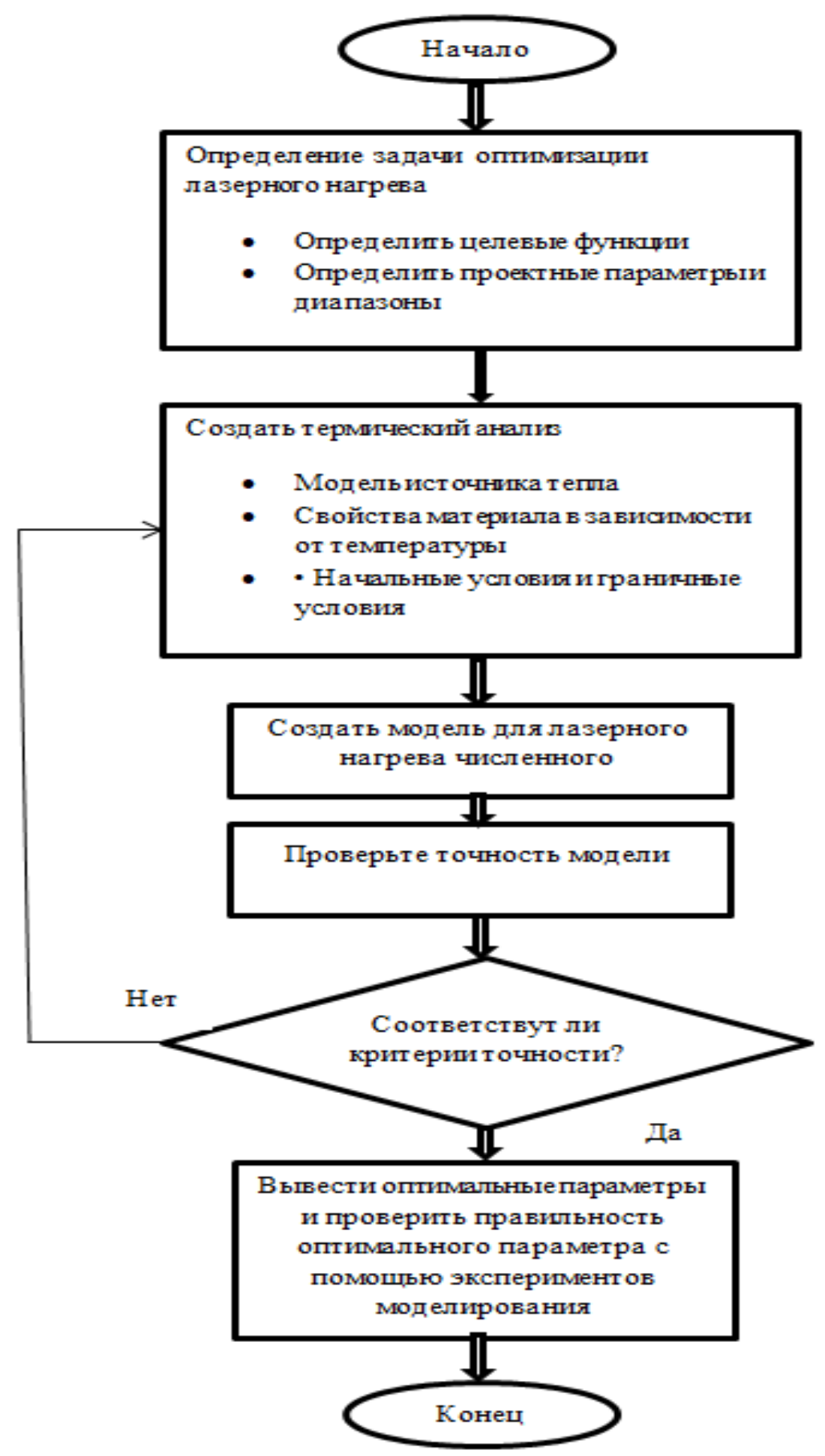




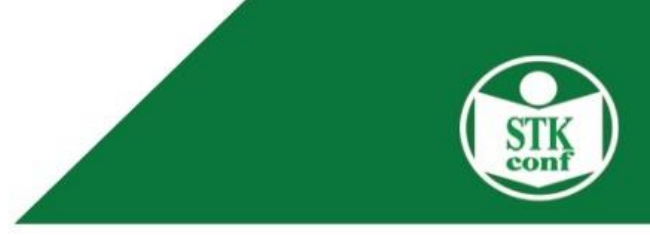

\section{5-17 March 2019}

\subsubsection{Virtual Specimen and Meshing}

The Laser heating was modeled over a virtual specimen by virtual Specimen and Meshing, 50.8 $\mathrm{mm}$ wide, $25.7 \mathrm{~mm}$ thick, this being the size of the plate in the experimental procedure for validation. For the purpose of improving the consistency of the model, the virtual specimen was divided into 1 domain, 8 boundaries, 18 edges, and 12 vertices of interest (Figure 3).As a consequence of this, mesh must be set along the laser path, whereas a coarser mesh is allowed for the purpose of reducing the simulation time: a triangle mesh of variable size was applied, accordingly, the parameter of element size is between $4.04 \mathrm{~mm}$ and $0.5 \mathrm{~mm}$. The maximum element size gain rate is 1.45 .

Initial and boundary conditions are given for domain. Generation $Q$ is provided along the processing path by laser heat; convection and radiation losses are operated at the over surface and convection and radiation losses are operated at each surface; a condition of thermal continuity is given with respect to domain for shared surface. The initial temperature of the domains is supposed as room temperature.

Figure 2.Triangular mesh of variable size on a virtual pattern.

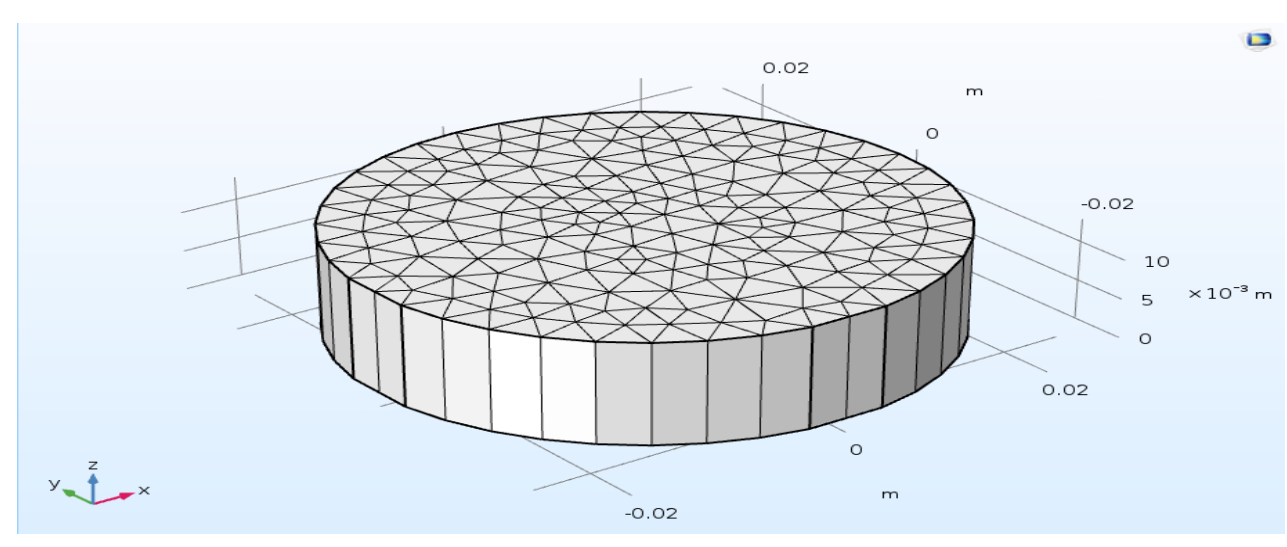

\subsection{Geometry of the Fusion Zone}

As the all element size of the fusion zone depends on the thermal properties, the model was centralized to be validated upon inspections in a transverse cross-section with the moving direction of the laser beam. The simulation depth of the fusion zone had to be conducted by the indirect measurement: a transverse plane at half-length was considered with respect to the traveling direction. As expected, any increase in the investigated peak temperature produced a proportional increase in the expanse of the fusion zone. 


\section{3rd International Conference}

\section{On Applied Research In SCIENCE, TECHNOLOGY \& KNOWLEDGE}

\section{5-17 March 2019}

Figure 3: Contour lines to determine the extent of the merge zone for the processing condition.



According to solidify range of the alloy, the temperature zone was decided by melting. In fact, as $715 \mathrm{~K}$ is the limit of the solidify range, at any point above this temperature limit was occurred by melting. For each given loading condition, the temperature was compared with the corresponding actual geometry (Table 1 ). 


\section{3rd International Conference}

\section{On Applied Research In SCIENCE, TECHNOLOGY \& KNOWLEDGE}

\section{5-17 March 2019}

Table 1: Comparison of different temperatures on the metal

\begin{tabular}{|l|l|l|l|l|}
\hline Time $(\mathrm{s})$ & Temperature $(\mathrm{K})$, Domain Probe 1 & Temperature $(\mathrm{K})$, Domain Probe 2 & Temperature (K), Domain Probe 3 & T_max-T_min (K) \\
\hline 58.531 & 552.95 & 682.10 & 518.49 & 163.62 \\
\hline 58.634 & 552.96 & 688.67 & 518.10 & 170.57 \\
\hline 58.736 & 552.99 & 686.91 & 517.71 & 169.20 \\
\hline 58.828 & 553.01 & 691.69 & 517.38 & 174.30 \\
\hline 58.920 & 553.03 & 685.50 & 517.06 & 168.44 \\
\hline 59.012 & 553.05 & 687.49 & 516.76 & 170.74 \\
\hline 59.094 & 553.07 & 690.40 & 516.48 & 173.93 \\
\hline 59.177 & 553.07 & 692.28 & 516.22 & 176.06 \\
\hline 59.260 & 553.08 & 697.86 & 515.97 & 181.89 \\
\hline 59.335 & 553.07 & 701.48 & 515.75 & 185.73 \\
\hline 59.409 & 553.07 & 696.39 & 515.55 & 180.85 \\
\hline 59.484 & 553.06 & 706.63 & 515.34 & 191.29 \\
\hline 59.558 & 553.06 & 718.41 & 515.15 & 203.26 \\
\hline 59.633 & 553.03 & 731.15 & 514.97 & 216.18 \\
\hline 59.707 & 552.97 & 538.21 & 223.41 \\
\hline 59.782 & 552.82 & 750.15 & 514.79 & 235.51 \\
\hline 59.849 & 552.57 & 747.59 & 514.49 & 233.10 \\
\hline 59.916 & 552.20 & 738.37 & 514.36 & 224.00 \\
\hline 59.983 & 551.68 & 717.84 & 514.24 & 203.60 \\
\hline 60.050 & 551.17 & 712.97 & 514.11 & 198.86 \\
\hline
\end{tabular}

Figure 4: Maximum, minimum and average temperatures as a function of time. 


\section{5-17 March 2019}

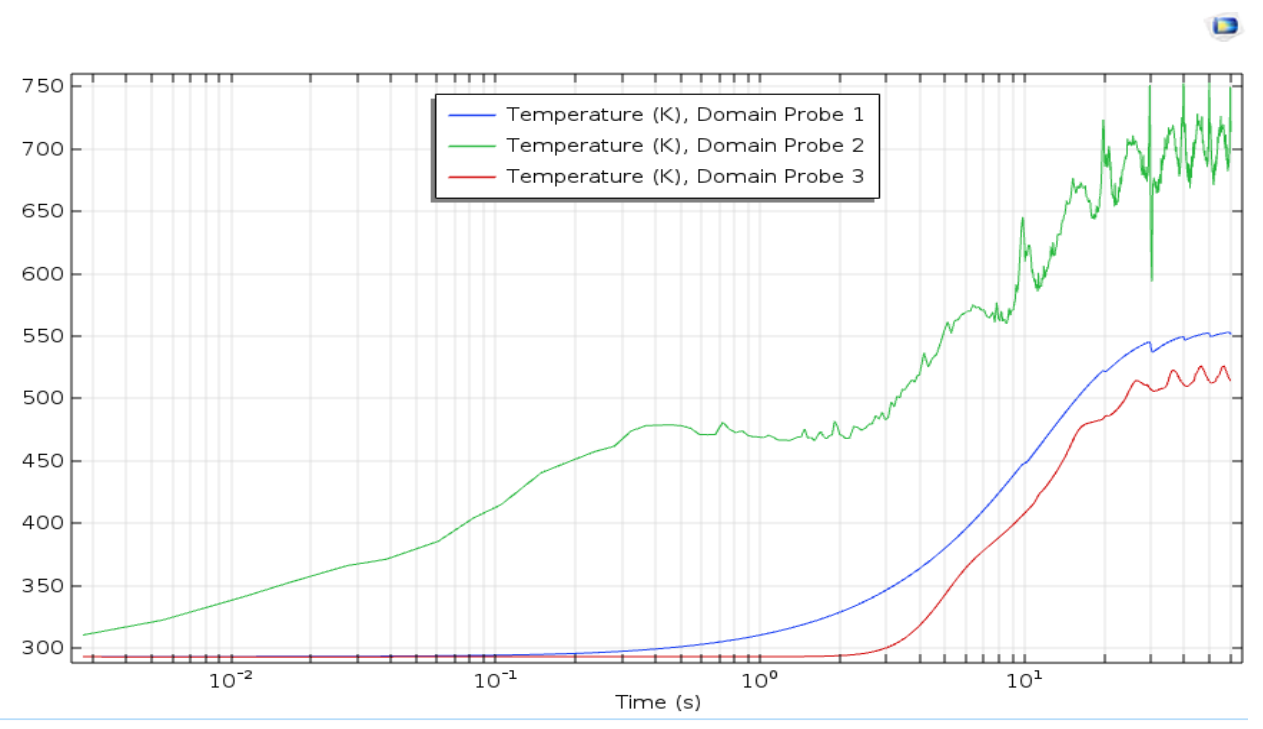

The temperature response at time (max, avg and $\min$ ) is show in Fig. For the numerical simulation of this experiment, the temperature under the substrate was extracted after simulating heat transfer through powder. The results demonstrate that the maximum temperature laser exposure powder within $0.1 \mathrm{~s}$ and the average temperature within $10 \mathrm{~s}$ and the minimum temperature $14 \mathrm{~s}$ in $450{ }^{\circ} \mathrm{C}$, respectively.

\section{Conclusions}

The purpose of this research is parameter control of laser heating process. To summarize, results could be divided into three groups, temperature distribution, heat transfer and heating rate. Two parameters and their interaction have a strong influence on the laser process: power and laser scanning speed. The study of influence of laser parameters helps to find the best laser parameter setting to improve the effective of materials. This modeling can be helpful to define the range of real laser parameters for other material, especially difficult to machine materials.

\section{References}




\section{5-17 March 2019}

[1] Yan, J., MAsoudi, N., Battiato, I, and Fadel, G, 2015, “ Optimization of Process Parameters in Laser Engineered Net Shaping (LENS) Deposition of Multi-Materials," Proceedings of the ASME 2015 International Design Engineering technical Conference \& Computer and Information in Engineering Conference (IDETC/CIE), Boston, Massachusetts, August 2015. [2] Antipas, G. S. E, 2015, "Experimental and Fust Principles Assesment of Plasma Attenuation during Laser Treatment of an Alloy," Transactions of the IMF, Vol. 93(1), pp. 5356.

[3] M. Masoomi, E. Alaa, N. Shamsaei and S. Thompson, "Modeling, simulation and experimental validation of heat transfer during selective laser melting," in Proceedings of the ASME 2015 International Mechanical Engineering Congress \& Exposition, Houston, 2015. 